\title{
Expression of aquaporin 5 in primary carcinoma and lymph node metastatic carcinoma of non-small cell lung cancer
}

\author{
TIANHE SONG ${ }^{1}$, HONG YANG ${ }^{2}$, JAMES CHUNG MAN HO ${ }^{3}$, SYDNEY CHI WAI TANG $^{3}$, \\ STEPHEN CHO WING SZE ${ }^{1}$, LIXING LAO ${ }^{1}$, YING WANG ${ }^{2}$ and KALIN YANBO ZHANG ${ }^{1}$

\begin{abstract}
${ }^{1}$ School of Chinese Medicine, LKS Faculty of Medicine, The University of Hong Kong, Pokfulam 999077, Hong Kong, SAR;
${ }^{2}$ Department of Histopathology, Sichuan Cancer Hospital, Chengdu, Sichuan 610041; ${ }^{3}$ Department of Medicine,

LKS Faculty of Medicine, The University of Hong Kong, Pokfulam 999077, Hong Kong, SAR, P.R. China
\end{abstract}

Received June 9, 2014; Accepted February 26, 2015

DOI: $10.3892 / \mathrm{ol} .2015 .3108$

\begin{abstract}
Aquaporin 5 (AQP5), a water channel protein, is highly expressed in non-small cell lung cancer (NSCLC) tissues compared with adjacent normal tissues. AQP5 expression in lung cancer tissues is associated with a poor prognosis. The present study aimed to analyze the expression of AQP5 and investigate its role in primary and lymph node metastatic NSCLCs. An immunohistochemical labeled streptavidin-biotin method was used to determine the expression of AQP5 in 94 cases of NSCLC primary carcinoma, which included 51 cases accompanied by lymph node metastasis. The results revealed that the expression of AQP5 was significantly higher in adenocarcinomas compared with squamous cell carcinomas $(\mathrm{P}=0.002)$. In addition, the percentage of AQP5 expression in the primary carcinomas with lymph node metastasis was significantly higher compared with those without lymph node metastasis $(\mathrm{P}=0.024)$. However, no statistically significant difference in the percentage of AQP5 expression was observed between the metastatic and the primary carcinomas $(\mathrm{P}=0.377)$. The expression of AQP5 exhibited a correlation with the tumor-node-metastasis staging of NSCLC $(\mathrm{P}=0.027)$. The percentage of AQP5 expression in stage III and IV tumors was higher than that in stage I and II tumors. In addition, AQP5 expression was correlated with the survival rate of NSCLC patients $(\mathrm{P}=0.051)$. In conclusion, the results of the present study provide evidence for the AQP5-facilitated incidence, progression and metastasis of NSCLC.
\end{abstract}

Correspondence to: Dr Kalin Yanbo Zhang, School of Chinese Medicine, LKS Faculty of Medicine, The University of Hong Kong, 10 Sassoon Road, Pokfulam 999077, Hong Kong, SAR, P.R. China E-mail: zhang.yanbo@yahoo.com

Professor Ying Wang, Department of Histopathology, Sichuan Cancer Hospital, 55 Renmin South Road 4th Section, Chengdu, Sichuan 610041, P.R. China

E-mail: wangyingmaria@163.com

Key words: non-small cell lung cancer, aquaporin 5, primary carcinoma, lymph node metastatic carcinoma, metastasis
Therefore, AQP5 may be used as a potential target to investigate the incidence, progression and metastasis of NSCLC.

\section{Introduction}

Lung cancer is the leading cause of cancer-associated mortalities in males and females worldwide $(1,2)$. The two primary types of lung cancer, small cell lung cancer and non-small cell lung cancer (NSCLC), account for $90 \%$ of all the diagnosed lung cases. NSCLC, which includes squamous cell carcinoma, adenocarcinoma and large cell carcinoma, constitutes $\sim 80 \%$ of all lung cancer types (3-5). The incidence, progression and underlying mechanisms of tumor metastasis have been a focus of investigation. Although advances have been made in the treatment and diagnosis of NSCLC over the last several years, the overall prognoses for patients with NSCLC remain poor (6). A number of studies have attempted to identify novel prognostic and predictive biomarkers for the diagnosis and treatment of NSCLC $(7,8)$.

Previous studies have established that tumor growth, development, invasion and metastasis depend upon sufficient nutrient supply and metabolism, and that water molecules are important in the modulation of the tumor microenvironment and metabolism $(9,10)$. Increasing evidence has demonstrated that aquaporins (AQPs), a family of small transmembrane proteins responsible for water transport, are involved in the incidence and progression of cancer (11-13). In particular, AQP1 has been found to be implicated in the incidence and progression of tumors $(11,12)$, whilst AQP5 has been proposed to enhance tumor growth and be associated with tumor metastasis (13).

AQPs are widely distributed in a variety of organs, and primarily regulate the transport of water molecules by osmotic pressure. In addition, AQPs regulate the transmembrane transport of other small molecules, including glycerol and urea. In total, 12 types of AQPs are expressed in humans, and AQP1, 3, 4 and 5 are expressed in the respiratory system. AQP5 is primarily distributed in the epithelial cells of type I alveoli and glandular epithelial cells of the submucosal glands. Its main functions are water transport and involvement in glandular secretion and airway hyperactivity (14). Previous studies have established that AQP1, 3 and 5 are associated 
with malignant tumors. Furthermore, it has been demonstrated that AQP1 and 3 promote the metastasis of tumors $(15,16)$. Woo et al (13) revealed that AQP5 enhanced the proliferation of tumor cells. In addition, high AQP5 expression has been identified to be associated with the metastasis of rectal, uterine and ovarian cancer (17-19).

However, only a limited number of studies have investigated the role of AQP5 in cancer. The aims of the present study were to investigate the expression of AQP5 and determine its role in NSCLC.

\section{Patients and methods}

Study participants. In total, 145 patients were selected by Professor Ying Wang from the Sichuan Cancer Hospital (Chengdu, China) between August 2006 and February 2008. The patients were recruited through oncologist referrals, having been diagnosed with NSCLC following presentation with lung cancer symptoms, such as chest pain, shortness of breath, hemoptysis and wheezing. The inclusion criteria were as follows: Patients with NSCLC; patients treated with surgery; and patients agreed to participate the present study. All the patients provided written informed consent for participation. The study experiments conformed to the ethical guidelines of the 1975 Declaration of Helsinki (20), and were approved by the Institutional Review Board of the Sichuan Cancer Hospital.

Of the 145 NSCLC patients, 51 patients were excluded due to having received prior radiation therapy or chemotherapy, having incomplete clinical information, or unavailability of paraffin-embedded tissue sections. The remaining 94 patients with primary NSCLC (male, 68; female, 26; age range, 29-82 years) were included in the present study (Fig. 1). These patients had not received prior radiation therapy or chemotherapy. Complete clinical information and paraffin-embedded sections were obtained. The pathological staging of the histological types was based on the standard histological classification of lung cancer, established by the World Health Organization in 2004 (21). Following the collection of clinical samples for the present study, the presence or absence of metastasis was assessed.

Patient follow-up. The patients were routinely followed-up on an outpatient basis, according to a standard protocol. Briefly, in the first year following treatment/surgery, visits were conducted every 3 months; in the second and third years, every 6 months; and until the end of the fifth year, once every 12 months. Patients who did not attend the outpatient program were contacted by telephone and/or letter in order to obtain follow-up data, until the visit cut-off date on August 30, 2012. The rate of missed visits was $4.6 \%$. The period of survival was calculated from the date of diagnosis to the date of the final follow-up or the date of mortality due to recurrence or metastasis.

Immunohistochemical staining. AQP5 expression in the biopsy tissue specimens was detected by immunohistochemical analysis. The biopsy tissue specimens were collected from the NSCLC patients who had undergone surgical removal of the tumor. Tumor sections measuring $5 \mu \mathrm{m}$ in thickness were initially deparaffinized and rehydrated in a graded series of alcohols: Slides were submerged in xylene two times for $5 \mathrm{~min}$, before soaking in $100 \%$ ethanol two times for $5 \mathrm{~min}, 95 \%$ ethanol for $5 \mathrm{~min}$ and in $80 \%$ ethanol for $5 \mathrm{~min}$. Following rehydration, an antigen retrieval process was performed under high pressure (22). The sections were then washed twice in phosphate-buffered saline (PBS)-Tween 20 for $2 \mathrm{~min}$ each. The sections were allowed to cool prior to blocking with $20 \%$ goat serum in PBS for $15 \mathrm{~min}$. Next, the sections were incubated at $37^{\circ} \mathrm{C}$ for $1 \mathrm{~h}$, and then overnight at $4^{\circ} \mathrm{C}$ with a polyclonal rabbit antibody against AQP5 (dilution, 1:100; Santa Cruz Biotechnology, Inc., Dallas, TX, USA; catalog no. SC-28628). Subsequent to washing three times with PBS-Tween 20 for 2 min each, the sections were incubated for $10 \mathrm{~min}$ at room temperature in a peroxidase blocking solution. Next, the sections were rinsed three times with PBS-Tween 20 for 2 min each and incubated with a biotinylated goat anti-rabbit IgG secondary antibody (dilution, 1:100; Santa Cruz Biotechnology, Inc.; catalog no. SC-2040) in PBS. Subsequent to washing, the sections were incubated in a horseradish peroxidase-streptavidin solution (dilution, 1:100; Sigma-Aldrich, St. Louis, MO, USA) for $30 \mathrm{~min}$ at $37^{\circ} \mathrm{C}$. The sections were again rinsed three times in PBS-Tween 20 for 2 min each and incubated in a peroxidase substrate solution (Sigma-Aldrich). Subsequently, the sections were rinsed in running tap water for 2-5 min, followed by dehydration with $95 \%$ ethanol for $1 \mathrm{~min}$ and twice with $100 \%$ ethanol for 3 min each. The sections were then cleared twice in $100 \%$ xylene for $5 \mathrm{~min}$ each and then covered with neutral gum (Bioworld Technology, Inc., St. Louis Park, MN, USA). The primary and secondary antibodies and 3,3'diaminobenzidine tetrahydrochloride (DAB) were obtained from Santa Cruz Biotechnology, Inc. All the steps were treated with deoxyribonuclease (Life Technologies, Grand lsland, NY, USA). This method was used to detect the expression of AQP5 in the lung cancer tissues. Microscopic immunohistochemical analysis of the tissue sections was performed using an Olympus BX41 microscope (Olympus Corporation, Tokyo, Japan).

Scoring of immunohistochemical staining results. Scoring of the AQP5 expression levels and analysis of all the specimens were performed by technicians who were blinded to the clinical information. The appearance of yellowish-brown granules in the cytoplasm was regarded to be a positive result for the expression of AQP5. The staining intensity of the AQP5-positive cells was scored based on the percentage of stained tumor cells as follows: i) 0 , unstained; ii) 1 , pale yellow staining; iii) 2 , yellowish-brown staining; and iv) 3 , brown staining. Scores were also assigned according to the percentage of AQP5-positive cells as follows: i) $4,>75 \%$; ii) $3,51-75 \%$; iii) $2,11-50 \%$; iv) $1, \leq 10 \%$; and v) 0 , negative. The two scores obtained for each case were then multiplied and assigned to one of the following groups: i) $0-2,(-)$; ii) 3-5, (+); iii) 5-8, (++); and iv) $9-12,(+++)(23)$.

Statistical analysis. To avoid bias, sample collection and data analysis were conducted without knowledge of the subject status. SPSS version 15.0 statistical software (SPSS, Inc., Chicago, IL, USA) was used for data analysis. The $\chi^{2}$ test was used to compare differences in AQP5 expression, the Kaplan-Meier method was used for the analysis of the survival 


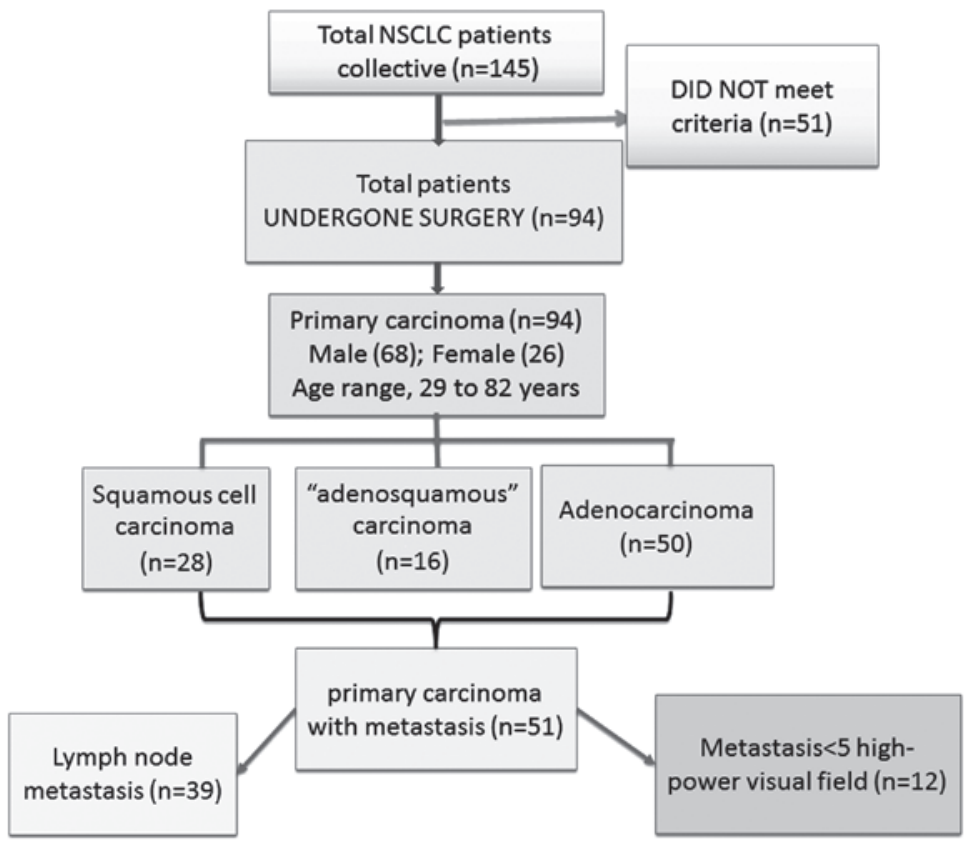

Figure 1. Study population. Flowchart revealing the composition of the patient collective included in the present study. NSCLC, non-small cell lung cancer.

Table I. Clinicopathological characteristics of NSCLC patients ( $\mathrm{n}=94)$.

\section{Clinicopathological characteristics}

\section{Gender}

Male

Female

Histological type

Squamous cell carcinoma

Adenocarcinoma

Adenosquamous carcinoma

Differentiation $^{\mathrm{a}}$

Well

Moderate

Poor

TNM staging

$\begin{array}{ll}\text { I } & 15(16.0) \\ \text { II } & 33(35.1) \\ \text { III } & 36(38.3) \\ \text { IV } & 10(10.6)\end{array}$

${ }^{\mathrm{a}} 16$ cases could not be staged. NSCLC, non-small cell lung cancer; TNM, tumor-node-metastasis.

data and the log-rank test was used to compare the survival curves. $\mathrm{P}<0.05$ was considered to indicate a statistically significant difference.

\section{Results}

Study population and baseline data. Fig. 1 shows the screening, randomization and participant flow of the different groups. Of
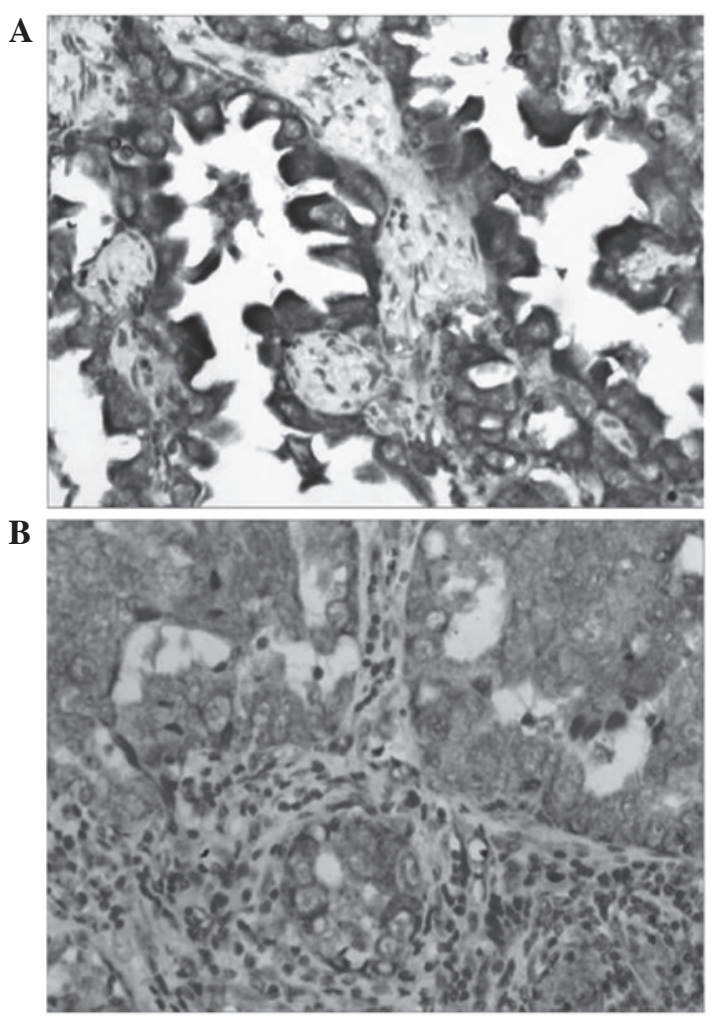

Figure 2. Aquaporin 5 expression in (A) adenocarcinoma and (B) lymph node metastasis (labeled streptavidin biotin staining; magnification, x200).

the 145 randomly selected patients, 94 were found to conform to the selection criteria. Of the 94 cases of NSCLC (male, 68; female, 26; mean age, 58.68 years; age range, 29-82 years), 28 presented with squamous cell carcinoma, 16 with adenosquamous carcinoma and 50 with adenocarcinoma (Table I). Overall, there were 15 cases of highly-differentiated carcinoma, 33 cases of moderately-differentiated carcinoma, 
Table II. AQP5 expression in different pathological tissues.

\begin{tabular}{|c|c|c|c|c|c|c|c|}
\hline \multirow[b]{2}{*}{ Pathological features } & \multirow[b]{2}{*}{$\mathrm{n}$} & \multicolumn{4}{|c|}{ AQP5 expression } & \multirow[b]{2}{*}{$\chi^{2}$} & \multirow[b]{2}{*}{ P-value } \\
\hline & & - & + & ++ & +++ & & \\
\hline \multicolumn{8}{|l|}{ Histological type } \\
\hline Squamous cell carcinoma & 28 & 20 & 5 & 2 & 1 & 14.38 & 0.002 \\
\hline Adenocarcinoma & 50 & 16 & 8 & 14 & 12 & & \\
\hline Adenosquamous carcinoma & 16 & 4 & 3 & 6 & 3 & & \\
\hline \multicolumn{8}{|l|}{ Differentiation $^{\mathrm{a}}$} \\
\hline Well & 15 & 8 & 3 & 2 & 2 & 2.16 & 0.904 \\
\hline Moderate & 33 & 16 & 6 & 6 & 5 & & \\
\hline Poor & 30 & 12 & 4 & 8 & 6 & & \\
\hline \multicolumn{8}{|l|}{ TNM staging } \\
\hline I & 15 & 11 & 1 & 2 & 1 & 18.73 & 0.027 \\
\hline II & 33 & 14 & 5 & 9 & 5 & & \\
\hline III & 36 & 16 & 5 & 4 & 11 & & \\
\hline IV & 10 & 1 & 3 & 5 & 1 & & \\
\hline
\end{tabular}

${ }^{\mathrm{a}} 16$ cases could not be staged. AQP5, aquaporin 5; TNM, tumor-node-metastasis.

Table III. AQP5 expression in primary and metastatic carcinomas.

\begin{tabular}{|c|c|c|c|c|c|c|c|}
\hline \multirow[b]{2}{*}{ Groups } & \multirow[b]{2}{*}{$\mathrm{n}$} & \multicolumn{4}{|c|}{ AQP5 expression } & \multirow[b]{2}{*}{$\chi^{2}$} & \multirow[b]{2}{*}{ P-value } \\
\hline & & - & + & ++ & +++ & & \\
\hline \multicolumn{8}{|l|}{ Lymph node metastasis } \\
\hline No & 43 & 25 & 6 & 9 & 3 & 9.40 & 0.024 \\
\hline Yes & 51 & 17 & 8 & 11 & 15 & & \\
\hline \multicolumn{8}{|l|}{ Tumor type } \\
\hline Primary carcinoma & 39 & 16 & 7 & 5 & 11 & 3.09 & 0.377 \\
\hline Metastatic carcinoma & 39 & 11 & 5 & 10 & 13 & & \\
\hline
\end{tabular}

AQP5, aquaporin 5 .

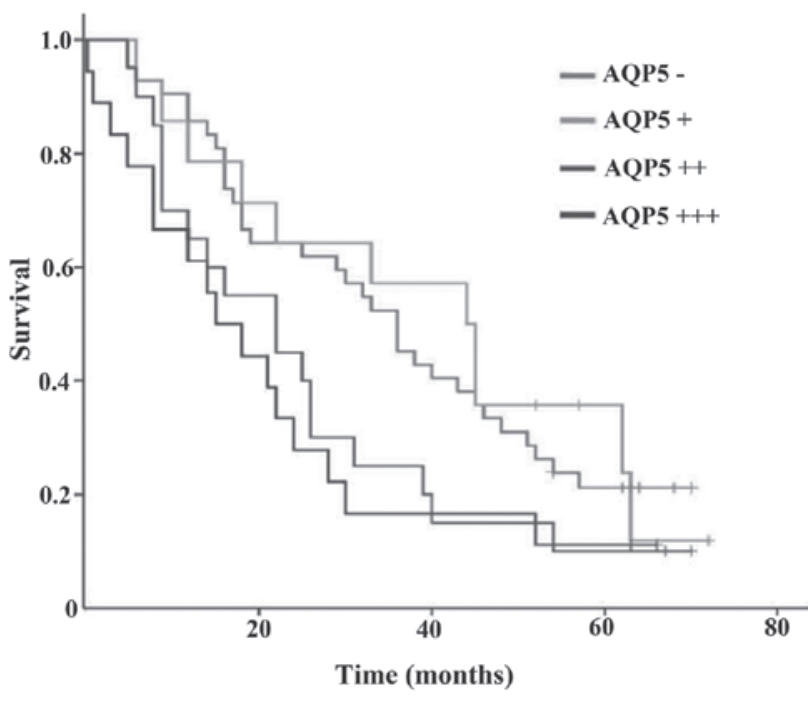

Figure 3. Survival curves of non-small cell lung cancer patients with different expression levels of AQP5. AQP5, aquaporin 5.
30 cases of poorly-differentiated carcinoma and 16 cases of adenosquamous carcinoma that could not be staged. In addition, 51 cases of primary carcinoma were accompanied by metastasis, including 39 cases with lymph node-specific metastasis (requirement for metastasis, $\geq 5 \mathrm{x}$ high-power visual field). In accordance with the revised standard for the 2009 lung cancer international tumor-node-metastasis (TNM) staging (24), 15 cases were at stage I, 33 cases were at stage II, 36 cases were at stage III and 10 cases were at stage IV (Table I). Patients with stages III and IV NSCLC did not undergo surgical treatment.

Association between AQP5 expression percentage and the clinical pathological features of NSCLC. AQP5 expression was associated with the histological type and TNM staging of the NSCLCs. The percentage of AQP5 expression in the adenocarcinomas was significantly higher compared with that in the squamous carcinomas $(\mathrm{P}=0.002)$. Of the 16 cases of adenosquamous carcinoma, 11 were positive for the 
expression of AQP5, which yielded an expression percentage of $68.8 \%$. This was significantly higher than the $31.2 \%$ expression percentage (5 cases) of the squamous carcinoma group $(\mathrm{P}<0.034)$. The positive expression percentages of AQP5 in stages III and IV NSCLCs were significantly higher compared with those in stages I and II NSCLCs $(\mathrm{P}=0.027)$. The expression percentages of AQP5 in the highly-, moderately- and poorly-differentiated tumors were 46.7, 51.5 and $60.0 \%$, respectively, and no statistically significant differences were observed $(\mathrm{P}=0.904$; Table II).

Association between AQP5 expression and NSCLC metastasis. The AQP5 expression percentages in primary carcinomas accompanied by lymph node metastasis were significantly higher compared with those in primary carcinomas not accompanied by lymph node metastasis $(\mathrm{P}=0.024)$. The AQP5 expression percentages in metastatic carcinoma cases did not exhibit a statistically significant difference compared with the primary carcinoma cases $(\mathrm{P}=0.377$; Table III). In primary carcinoma cases with high AQP5 expression, the metastatic carcinomas exhibited augmented AQP5 expression. Therefore, AQP5 may be involved in the metastasis of NSCLC. In addition, the results indicated that NSCLCs with elevated AQP5 expression are more likely to be associated with lymph node metastasis.

Association between AQP5 expression and survival rate of NSCLC patients. AQP5 expression was evaluated in tissue samples by immunohistochemical analysis. The appearance of yellowish-brown granules in the cytoplasm was regarded as a positive result for the expression of AQP5 (Fig. 2). The staining intensity of the AQP5-positive cells was scored, as well as the percentage of AQP5-positive cells. The two scores obtained for each case were multiplied and assigned to one of the following groups: $(-),(+),(++)$, and $(+++)$. The results of the present study revealed that the five-year survival rate of NSCLC patients with scores of $(-),(+),(++)$, and $(+++)$ were $21.43,28.57,10.00$ and $11.11 \%$, respectively. The log-rank test demonstrated a clear association between the expression of AQP5 and the survival rate of NSCLC patients $(\mathrm{P}=0.051$; Fig. 3). An upregulation in the expression of AQP5 was correlated with a decrease in the survival rate of NSCLC patients.

\section{Discussion}

The present study examined the expression of AQP5 in 94 cases of NSCLC primary carcinoma and 51 cases of metastatic carcinoma. The results revealed that AQP5 expression in NSCLCs was associated with lymph mode metastasis. AQP5 expression in NSCLC primary carcinomas accompanied by lymph node metastasis was markedly higher compared with those without lymph node metastasis. When the AQP5 expression levels in primary carcinomas was compared with that in metastatic carcinomas, no statistically significant difference was identified. In cases of primary carcinoma with high AQP5 expression, the metastatic carcinomas also exhibited augmented AQP5 expression. This indicated that NSCLCs with elevated AQP5 expression levels are more likely to be associated with lymph node metastasis, and that AQP5 plays an important role in the metastasis of
NSCLCs. In addition, the present study revealed that AQP5 expression was associated with the NSCLC clinical stage. The expression levels of AQP5 in stage III and IV tumors were significantly higher compared with stage I and II tumors. This observation indicated that a high level of AQP5 was associated with the promotion of lung cancer metastasis. Furthermore, AQP5 was revealed to be closely associated with the incidence and progression of adenocarcinomas. The positive expression percentage of AQP5 in adenocarcinomas was $68.0 \%$, which was markedly higher than the value of $28.6 \%$ in squamous carcinomas. In the 16 cases of squamous carcinoma, the positive expression percentage of AQP5 in adenocarcinomas was $68.8 \%$, which was notably higher than the value of $31.2 \%$ in squamous carcinomas. These results indicate an association between AQP5 and adenocarcinomas and a role for AQP5 in the incidence and progression of adenocarcinomas. Whether the elevated expression levels of AQP5 in adenocarcinomas can be used as an indirect marker during clinical and pathological diagnosis requires further investigation. In addition, the mechanisms involved in the promotion of tumor metastasis are under investigation. AQP5 phosphorylation has been demonstrated to be an important event involved in the promotion, incidence and progression of tumors (13). Previous studies have established that AQP5 can activate the RAS/ERK/RB pathway in rectal cancer cells and enhance the incidence and progression of cancer. Furthermore, an association with the metastasis of rectal cancer was also identified $(17,25)$.

In conclusion, the present study revealed an association between high AQP5 expression and the high invasiveness and metastatic percentage of NSCLC, as well as with the incidence and progression of adenocarcinomas. Histopathological analysis revealed an association between AQP5 and the metastasis, incidence and progression of NSCLC.

\section{References}

1. Jemal A, Siegel R, Ward E, Murray T, Xu J and Thun MJ: Cancer statistics, 2007. CA Cancer J Clin 57: 43-66, 2007.

2. Stewart BW and Wild CP (eds): Word Cancer Report 2014. IARC Nonserial Publication, 2014.

3. Esposito L, Conti D, Ailavajhala R, Khalil N and Giordano A: Lung cancer: Are we up to the challenge? Curr Genomics 11: 513-518, 2010.

4. Maziak DE, Marman BR, MacKay JA and Evans WK; Cancer Care Ontario Practice Guidelines Initiative Lung Cancer Disease Site Group: Photodynamic therapy in non-small cell lung cancer: a systematic review. Ann Thorac Surg 77: 1484-1491, 2004.

5. Hoffman PC, Mauer AM and Vokes EE: Lung cancer. Lancet 355: 479-485, 2000.

6. Schreiber G and McCrory DC: Performance characteristics of different modalities for diagnosis of suspected lung cancer: summary of published evidence. Chest 123 (Suppl 1): 115S-128S, 2003.

7. Aggarwal C, Somaiah N and Simon GR: Biomarkers with predictive and prognostic function in non-small cell lung cancer: ready for prime time? J Natl Compr Canc Netw 8: 822-832, 2010.

8. Akhtar S, Meeran SM, Katiyar N and Katiyar SK: Grape seed proanthocyanidins inhibit the growth of human non-small cell lung cancer xenografts by targeting insulin-like growth factor binding protein-3, tumor cell proliferation and angiogenic factors. Clin Cancer Res 15: 821-831, 2009.

9. Wang W, Li Q, Yang T Bai G, Li D, Li Q and Sun H: Expression of AQP5 and AQP8 in human colorectal carcinoma and their clinical significance. World J Surg Oncol 10: 242, 2012.

10. Jung HJ, Park JY, Jeon HS and Kwon TH: Aquaporin-5: a marker protein for proliferation and migration of human breast cancer cells. PLoS ONE 6: e28492, 2011. 
11. Saadoun S, Papadopoulos MC, Hara-Chikuma $M$ and Verkman AS: Impairment of angiogenesis and cell migration by targeted aquaporin-1 gene disruption. Nature 434: 786-792, 2005.

12. Burghardt B, Elkaer ML, Kwon TH, Rácz GZ, Varga G, Steward MC and Nielsen S: Distribution of aquaporin water channels AQP1 and AQP5 in the ductal system of the human pancreas. Gut 52: 1008-1016, 2003.

13. Woo J, Lee J, Chae YK, Kim MS, Baek JH, Park JC, et al: Over expression of AQP5, a putative oncogene, promotes cell growth and transformation. Cancer Lett 264: 54-62, 2008.

14. Verkman AS: More than just water channels: unexpected cellular roles of aquaporins. J Cell Sci 118: 3225-3232, 2005.

15. Liu YL, Matsuzaki T, Nakazawa T, Murata S, Nakamura N, Kondo T, Iwashina M, Mochizuki K, Yamane T, Takata K and Katoh R: Expression of aquaporin 3 (AQP3) in normal and neoplastic lung tissues. Hum Pathol 38: 171-178, 2007.

16. Hoque MO, Soria JC, Woo J, Lee T, Lee J, Jang SJ, Upadhyay S, Trink B, Monitto C, Desmaze C, Mao L, Sidransky D and Moon C: Aquaporin 1 is overexpressed in lung cancer and stimulates NIH-3T3 cell proliferation and anchorage-independent growth. Am J Pathol 168: 1345-1353, 2006.

17. Kang SK, Chae YK, Woo J, Kim MS, Park JC, Lee J, Soria JC, Jang SJ, Sidransky D and Moon C: Role of human aquaporin 5 in colorectal carcinogenesis. Am J Pathol 173: 518-525, 2008.

18. Zhang T, Zhao C, Chen D and Zhou Z: Overexpression of AQP5 in cervical cancer: correlation with clinicopathological features and prognosis. Med Oncol 29: 1998-2004, 2012.
19. Yang JH, Shi YF, Cheng Q and Deng L: Expression and localization of aquaporin-5 in the epithelial ovarian tumors. Gynecol Oncol 100: 294-299, 2006.

20. Declaration of Helsinki, 29th World Medical Association General Assembly, Tokyo, Japan, 1975.

21. Travis WD, Brambilla E, Müller-Hermelink HK and Harris CC (eds): World Health Organization Classification of Tumours: Pathology and Genetics of Tumours of the Lung, Pleura, Thymus and Heart. IARCPress, Lyon, France, 2004.

22. Fowler CB, Cunningham RE, Waybright TJ, Blonder J, Veenstra TD, O'Leary TJ and Mason JT: Elevated hydrostatic pressure promotes protein recovery from formalin-fixed, paraffin-embedded tissue surrogates. Lab Invest 88: $185-195,2008$

23. Xu LZ and Yang WT: Standard for appraisal of results of immunohistochemical reactions. Zhong Guo Ai Zheng Za Zhi 6: 229-231, 1996 (In Chinese).

24. Goldstraw P and Crowley JJ; IASLC International Staging Project: The International Association for the Study of Lung Cancer International Staging Project on Lung Cancer. J Thorac Oncol 1: 281-286, 2006.

25. Woo J, Lee J, Kim MS, Jang SJ, Sidransy D and Moon C: The effect of aquaporin 5 overexpression on the Ras signaling pathway. Biochem Biophys Res Commun 367: 291-298, 2008. 